\title{
State Reconstruction from a Limited Number of Synchronized Phasor Measurements: Application to Voltage Instability Detection
}

\author{
Mevludin Glavic, Senior Member, IEEE
}

\author{
Thierry Van Cutsem, Fellow, IEEE
}

approach of voltage stability assessment with limited number of measurement devices was considered in the early work of [5]. This work suggested the measurement of a reduced system state vector by properly clustering voltage-coherent buses and considering all voltages within a cluster equal to the representative voltage measured at one of its buses. With the system state approximated in this way, it was suggested to apply a standard voltage stability test, f.i. based on singular value decomposition of the Jacobian matrix [5].

In the authors' opinion, a more promising approach consists of estimating or "reconstructing" the vector of bus voltages, from which an appropriate voltage instability indicator can be computed. Several such indicators have been proposed, as documented for instance in [6], [7], [8]. Among them, the sensitivities of the total reactive power generation to individual load reactive powers were found very suitable [1], [2].

This raises the issue of "reconstructing" coherent, timesynchronized bus voltages from the limited number of available PMU data, for use in the above mentioned indicators.

To address the unobservability issue, the works in [9], [10] introduced the concept of interpolation of states of unobservable buses. Using the bus admittance matrix, a matrix of interpolation coefficients is derived relating the unobservable voltages to the PMU measured or observable buses. Reference [11] introduced a hybrid power flow model that combines PMU measurement data and power flow equations to increase situational awareness at the system level. This method is based on observable island defined as a set of buses whose states can be directly computed from PMU measurements complemented with assumed power flow conditions. Reference [12] introduced the PMU morphed power flow approach. Starting from a solved power flow, it proceeds by matching this known solution to the small number of PMU measurements by solving a linear programming problem with known system values (some generator outputs, total load in some areas) treated as constants and unknown values (the other generator outputs the other area loads, etc.) treated as controls.

Recently the authors proposed in [13], [14] an approach which they called "state reconstruction", as it shares some features of this general class of problems [15]. State reconstruction leads to minimizing, under constraints, a weighted least square objective involving the available phasor measurements together with bus power pseudo-measurements relative to a reference state.

The objective of this paper is to demonstrate that the widearea sensitivity-based method of [1], [2] still performs well

\begin{abstract}
M. Glavic (glavic@montefiore.ulg.ac.be) is a visiting professor at the Dept. of Electrical Engineering and Computer Science (Montefiore Institute), University of Liège, Sart Tilman B37, B-4000 Liège, Belgium.

T. Van Cutsem (t.vancutsem@ulg.ac.be) is research director at the Fund for Scientific Research (FNRS) and adjunct professor at the Dept. of Electrical Engineering and Computer Science, University of Liège, Sart Tilman B37, B-4000 Liège, Belgium.

One way to cope with such a situation is to rely on schemes using measurements gathered at one or several strategic locations, such as transmission corridors and load centers [3]. An
\end{abstract}




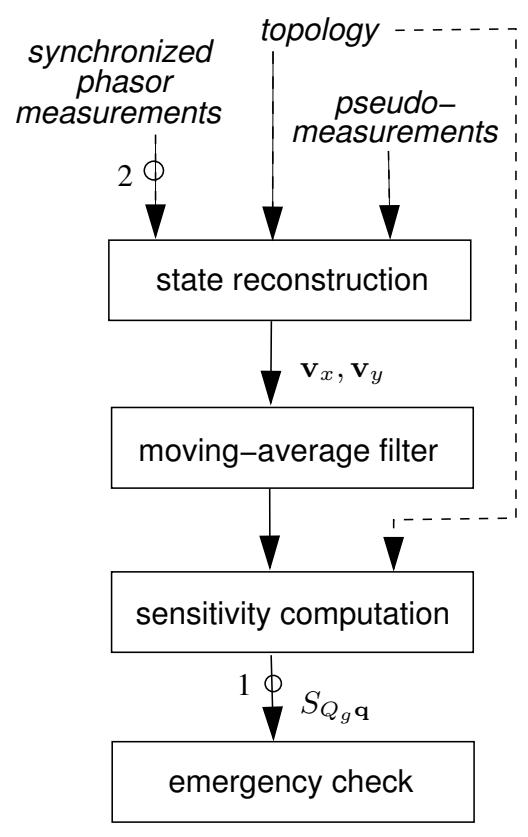

Fig. 1. Flowchart of procedure

when relying on bus voltages obtained from a state reconstruction procedure exploiting a limited number of synchronized phasor measurements which do not make the region of concern observable.

A flowchart of the whole procedure is given in Fig. 1.

The paper is organized as follows. Previous developments relative to voltage instability detection and state reconstruction are summarized in Sections II and III, respectively. The coupling of the two methods is discussed in Section IV. Section $\mathrm{V}$ presents simulation results on a 52-bus system with phasor measurements obtained from detailed time simulation of a long-term voltage unstable evolution. Finally, conclusions are offered in Section VI.

\section{OUTLINE OF WIDE-AREA VOLTAGE INSTABILITY DETECTION}

We outline hereafter the method detailed in [1], [2] for the early detection of a developing voltage instability from the bus voltages obtained from synchronized phasor measurements.

The method fits a set of algebraic equations $\varphi(\mathbf{z}, \mathbf{s})=\mathbf{0}$ to the sampled states, where $\mathbf{z}$ denotes the state vector and $\mathbf{s}$ is the vector of load active and reactive powers. These equations are obtained under the following assumptions:

- the network is represented by its bus admittance matrix, using real-time breaker status information;

- the short-term dynamics of generators, automatic voltage regulators, speed governors, static var compensators, etc. are not tracked but replaced by accurate equilibrium equations. This assumption is reasonable in so far as longterm voltage instability is of concern;

- the long-term dynamics driven by OverExcitation Limiters (OELs), Load Tap Changers (LTCs) and restorative loads are reflected through the change in measured voltages from one snapshot to the next;
- whether a generator is voltage controlled or field current limited is known or detected. Equations are adjusted accordingly, as recalled in the sequel.

Sensitivities are used to identify when a combination of load active and reactive powers has passed through a maximum, which is taken as indicator of emergency situation. Note that this requires knowing only the consumed powers: no information about load behaviour with voltage is needed. We consider the sensitivities of the total reactive power generation to individual load reactive powers. They are obtained from a general sensitivity formula [8] as:

$$
S_{Q_{g} \mathbf{q}}=-\boldsymbol{\varphi}_{\mathbf{q}}^{T}\left(\boldsymbol{\varphi}_{\mathbf{z}}^{T}\right)^{-1} \nabla_{\mathbf{z}} Q_{g}
$$

where $\mathbf{q}$ is the vector of load reactive powers, $\nabla_{\mathbf{z}} Q_{g}$ denotes the gradient of $Q_{g}$ with respect to the state vector $\mathbf{z}, \boldsymbol{\varphi}_{\mathbf{z}}$ is the Jacobian of $\boldsymbol{\varphi}$ with respect to $\mathbf{z}$, and $\varphi_{\mathrm{q}}$ the Jacobian of $\varphi$ with respect to $q$.

In theory these sensitivities change sign through infinity at the sought maximum load power point [1]. In practice, discontinuities as well as trajectory sampling generally prevent sensitivities from reaching very high values. What is sought is a sudden change in sign, i.e. we seek to identify a discrete time $k$ such that:

$$
S_{Q_{g} Q_{j}}(k-1)>d_{+} \quad \text { and } \quad S_{Q_{g} Q_{j}}(k)<d_{-}
$$

where $d_{+}>0$ and $d_{-}<0$ are thresholds. Note that the method does not require any other threshold tuning.

Computing $S_{Q_{g}}$ q merely requires solving one linear system with $\varphi_{\mathbf{z}}$ as matrix of coefficients and $\nabla_{\mathbf{z}} Q_{g}$ as independent term. The main computational effort lies in the factorization of $\varphi_{\mathrm{z}}$, for which efficient sparsity programming packages are available.

Attention must be paid to generator reactive power limits. An estimate of $E_{q}$, the e.m.f. proportional to field current, is used to identify whether a synchronous generator operates under control of its Automatic Voltage Regulator (AVR) or has been already limited by its OEL. Under AVR control, an equation such as:

$$
k E_{q}^{s}-G\left(V^{o}-V\right)=0
$$

is used, while under OEL control, it is replaced by an equation of the type:

$$
E_{q}-E_{q}^{l i m}=k E_{q}^{s}-E_{q}^{l i m}=0
$$

where $G$ is the open-loop static gain of the AVR, $E_{q}^{s}$ is the e.m.f. behind saturated synchronous reactances, $k$ is the saturation factor, $V$ is the terminal voltage, $V^{o}$ is the AVR voltage setpoint, and $E_{q}^{l i m}$ corresponds to the field current forced by the OEL. $E_{q}$ and $k$ are components of $\mathbf{z}$ together with the (rectangular components of) bus voltages and other variables.

Furthermore, it is of interest to anticipate the effect of an approaching OEL activation. To this purpose, when $E_{q}>$ $E_{q}^{\text {lim }}+\epsilon$, the OEL equation (4) is anticipatively substituted to the AVR equation (3) when evaluating the Jacobian $\varphi_{\mathbf{z}}$. This remains in effect as long as the OEL is acting, which is identified by $E_{q}^{l i m}-\epsilon \leq E_{q} \leq E_{q}^{l i m}+\epsilon$. In practice, since $E_{q}$ 
may undergo large but short-lasting changes under the effect of electromechanical transients, the inequality $E_{q}>E_{q}^{l i m}+\epsilon$ has to hold true for some period of time before the equation switching takes place.

As already mentioned, a requirement of the method is the availability of a PMU configuration making all bus voltages observable. Situations where the PMU configuration is not rich enough were partly tackled in [2], where it was shown that the Jacobians $\varphi_{\mathrm{z}}$ and $\varphi_{\mathrm{q}}$ can be limited to a subsystem including the region affected by voltage instability and the generators undergoing field current limitation. A simple handling of the voltages was considered at the boundary buses of the preserved sub-system. Although the sensitivities were somewhat affected by this approximation, their change in sign was still found to provide a reliable instability detection, showing that observability was needed only in the region subject to voltage instability [2].

This paper, however, focuses on more stringent (but more likely) situations with a significantly lower number of PMUs, not yielding observability of the region subject to voltage instability.

\section{OUTLINE OF STATE RECONSTRUCTION FROM LIMITED NUMBER OF PMUS}

The main purpose of state reconstruction is to track the changing system state and fill the gap between successive classical state estimations by exploiting the coherent, timesynchronized phasor measurements [13]. This could help monitoring the system after a large disturbance, in particular anticipating the near-future system evolution and possible cascading effects ${ }^{1}$.

The underlying ideas can be summarized as follows:

- the PMU configuration provides scarce measurements, which do not make the system observable. As a consequence, there is an infinite number of states satisfying the available set of synchronized measurements;

- we solve this indeterminacy by computing the state which yields the load powers closest (in a weighted Euclideannorm sense) to reference values;

- these reference values can be obtained either from the last estimate provided by a classical state estimator, as originally considered in [13], or from the last reconstructed state itself, as subsequently proposed in [14];

- the reference load power values are treated as pseudomeasurements, and complement the synchronized bus voltage and branch current measurements;

- both types of measurements are processed with proper weights in the least-square sense, together with sparse linear equality constraints relative to the network equations and zero injection information. The rectangular components of bus voltages and currents are taken as state variables.

To solve the indeterminacy left by the scarce PMUs, one could take bus voltages as reference, i.e. among all vectors

\footnotetext{
${ }^{1}$ state reconstruction could also replace a failing classical state estimation (owing to problems with bad data, divergence, etc.) or improve the accuracy of the latter when measurements suffer from bad data or time skew
}

of bus voltages which fit the phasor measurements, determine the one closest to reference voltages. The rationale behind the choice of load powers is as follows. A situation of interest for exploiting synchronized phasor measurements is when a disturbance takes place after the execution of the classical state estimator. In such a case, load powers change owing to their sensitivity to voltage and frequency. However, this change is usually in the order of a few percents, while complex bus voltages may change quite significantly.

Furthermore, we advocate the placement of PMUs at generator/compensator buses. Indeed, the powers produced by generators/compensators may also vary significantly under the effect of the disturbance, owing to their participation in voltage and/or frequency control ${ }^{2}$. Hence, state reconstruction is expected to be more accurate when information about the most changing variables is gathered through measurements.

The work in [14] extended the method of [13] by considering that the synchronized measurements, although scarce, can be redundant with the zero injections and/or the pseudomeasurements. Hence, they should not be treated as infinitely accurate or critical data. This leads to handling both the synchronized and pseudo-measurements in the following weighted least-square objective:

$$
\begin{aligned}
\min _{\mathbf{v}_{\mathbf{x}}, \mathbf{v}_{\mathbf{y}}, \mathbf{i}_{\mathbf{x}}, \mathbf{i}_{\mathbf{y}}} & \sum_{i=1}^{m} w_{i}\left(z_{i}-\mathbf{a}_{i}\left[\begin{array}{c}
\mathbf{v}_{\mathbf{x}} \\
\mathbf{v}_{\mathbf{y}} \\
\mathbf{i}_{\mathbf{x}} \\
\mathbf{i}_{\mathbf{y}}
\end{array}\right]\right)^{2} \\
& +\sum_{j=1}^{p} w_{P j}\left(P_{j}^{r e f}-v_{x j} i_{x j}-v_{y j} i_{y j}\right)^{2} \\
& +\sum_{j=1}^{p} w_{Q j}\left(Q_{j}^{r e f}-v_{y j} i_{x j}+v_{x j} i_{y j}\right)^{2}
\end{aligned}
$$

together with the following constraints:

$$
\begin{aligned}
\mathbf{G v}_{\mathbf{x}}-\mathbf{B v}_{\mathbf{y}}-\mathbf{i}_{\mathbf{x}} & =\mathbf{0} \\
\mathbf{B} \mathbf{v}_{\mathbf{x}}+\mathbf{G v}_{\mathbf{y}}-\mathbf{i}_{\mathbf{y}} & =\mathbf{0} \\
\mathbf{C}\left[\begin{array}{c}
\mathbf{i}_{\mathbf{x}} \\
\mathbf{i}_{\mathbf{y}}
\end{array}\right] & =\mathbf{0}
\end{aligned}
$$

In the objective function (5), $\mathbf{v}_{\mathbf{x}}, \mathbf{v}_{\mathbf{y}}, \mathbf{i}_{\mathbf{x}}, \mathbf{i}_{\mathbf{y}}$ are vectors of rectangular components of bus voltages and bus currents, respectively, $z_{i}$ is the $i$-th synchronized measurement of voltage or current component, $\mathbf{a}_{i}$ is a unit row vector with the nonzero entry corresponding to the measured variable, $m$ is the number of synchronized phasor measurements, $P_{j}^{r e f}$ and $Q_{j}^{r e f}$ are active and reactive power references, and $p$ is the number of pseudo-measurements.

Although they remain affected by sensor and communication channel errors [16], [17], synchronized measurements are expected to be more accurate than classical SCADA measurements. They are also expected to be more accurate than the pseudo-measurements. Hence, in (5), the weights $w_{i}$ assigned to phasor measurements should be larger than the weights $w_{P j}, w_{Q j}$ assigned to pseudo-measurements. Clearly,

\footnotetext{
${ }^{2}$ for instance, the outage of transmission or generation equipments is reflected in the reactive powers of nearby voltage controlled generators
} 
if a phasor measurement is critical, its corresponding weight does not matter.

The constraints $(6,7)$ are the network equations, where $\mathbf{G}$ is the real part of the bus admittance matrix and $\mathbf{B}$ the imaginary part. Finally, (8) relates to zero current mismatches at "transit" buses without load or generation connected. $\mathbf{C}$ is a simple matrix with 0 's and 1 's.

In the above optimization, the unknowns are $\mathbf{v}_{\mathbf{x}}, \mathbf{v}_{\mathbf{y}}, \mathbf{i}_{\mathbf{x}}$ and $i_{y}$. The objective is nonlinear but all constraints are linear.

\section{Voltage instability DETECTION FROM RECONSTRUCTED SYSTEM STATES}

The main idea of the approach reported in this paper is to keep the wide-area voltage instability detection method as proposed in [1], [2], but feed it with complex bus voltages obtained from state reconstruction (relying on scarce PMUs) instead of a state estimator (relying on PMUs making all bus voltages observable). By so doing, the accuracy, the generality and the important anticipation capability of the voltage instability detection method are preserved.

Moreover, the formulation that underlies the sensitivity computation [1] can be re-used with little additional effort for other purposes such as identifying the best location for remedial actions [18].

Although not specifically considered in the context of voltage instability detection, the PMU placement mentioned in the previous section perfectly fits the requirements of this task. Having PMUs placed at generator buses facilitates the tracking of their field currents, used in sensitivity analysis as indicated in Section II. Moreover, as recalled in the same section, the sensitivity analysis does not require load models, and hence does not require monitoring loads for the purpose of identifying those models. This is coherent with the use of mere pseudo-measurements instead of PMUs at load buses, for state reconstruction purposes. Finally, an additional advantage of locating PMUs at generator buses lies in the fact that they can serve the purposes of other applications such as assessing the damping of low-frequency oscillations, or tracking the system state between two classical state estimations.

In most cases, voltage instability is monitored in a region exposed to this risk due to a structural weakness. As recalled in Section II, sensitivity calculations can be limited to that region and its periphery, in order to limit the number of PMUs. With state reconstruction, the procedure is slightly different. References [13], [14] show that it is possible to accurately reconstruct the state of only a sub-network, placing PMUs on the generators located inside or at its periphery. Outside the region of interest, only pseudo-measurements may be used, of course at the expense of a lower accuracy. Thus, there is no need to partition the system and resort to an equivalent outside the region of interest. Instead, the Jacobian $\varphi_{\mathrm{z}}$ used in (1) can be computed for the whole system, relying on the whole vector of reconstructed bus voltages. Of course, all generators critical for voltage stability assessment should be included in the sub-network whose state is accurately reconstructed.

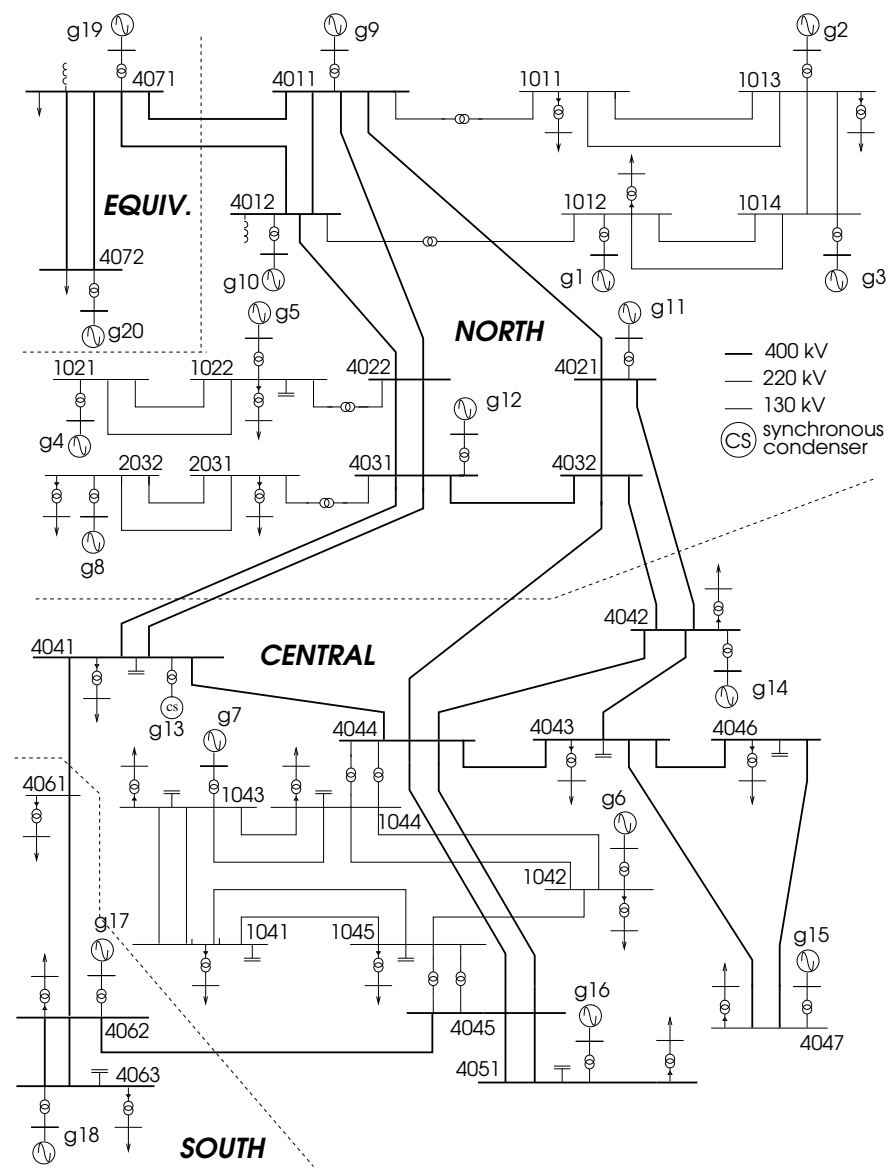

Fig. 2. Nordic32 test system

\section{Simulation Results}

\section{A. Test system}

This section provides illustrative results obtained with the Nordic32 test system previously used in [1], [2], [13], [14], [18]. The one-line diagram of this 52-bus, 20-machine system is shown in Fig. 2. The dynamic model of the system includes for each generator:

- a standard synchronous machine representation with 3 or 4 rotor windings;

- a generic model of hydro turbine and a simple model of speed governor, for generators in the North and Equiv areas (the other ones, not participating in frequency control, are represented with constant mechanical torque);

- a simple model of the excitation system, AVR and OEL.

Each load is represented by an exponential model with exponent 1 (constant current) for the active power and exponent 2 (constant admittance) for the reactive power. In addition, each load is fed through a transformer with automatic LTC. There is a delay of 30 seconds on the first tap change and a shorter delay on the subsequent steps, slightly different from one LTC to the other.

We consider the response to a 3-phase fault on line 40324044 , cleared by permanent opening of the line. The system is long-term voltage unstable under the effect of LTCs and OELs. 
The system response to this disturbance has been obtained with detailed time simulation, thus including the effect of short-term dynamics, such as electromechanical oscillations.

\section{B. Phasor and pseudo-measurements}

Phasor measurements have been assumed at selected locations, and the corresponding rectangular components of bus voltages and branch currents have been obtained by sampling the outputs of time simulation every $100 \mathrm{~ms}$. In some tests, measurement noise has been simulated by adding to each voltage or current component a random component with Gaussian probability distribution.

The accuracy of state reconstruction, and consequently of voltage instability detection, depends obviously on the configuration of available PMU measurements. Different configurations have been investigated. The results given hereafter have been obtained with :

- 5 PMUs: at buses g6, g7, g14, g15 and g16;

- 7 PMUs: at buses g6, g7, g11, g12, g14, g15 and g16.

Each PMU provides the complex voltage at the generator bus as well as the complex current injected by the generator. Each PMU is placed at the generator bus instead of the high-voltage end of the step-up transformer because, in this small test system, the latter choice would provide too good a coverage of the region and, hence, optimistic results.

In the 7-PMU configuration, for instance, active and reactive power injection pseudo-measurements are considered at buses: 1011, 1012, 1013, 1022, 1041, 1042, 1043, 1044, 1045, 2031, 2032, 4012, 4041, 4042, 4043, 4046, 4047, 4051, 4061, 4062, 4063, 4071, 4072, g1, g2, g3, g4, g5, g8, g9, g10, g13, g17, g18, g19 and g20. Note that in the 5-PMU configuration, buses g11 and g12 are also provided with a power injection pseudomeasurement, in replacement for the PMU. No distribution bus is involved in the state reconstruction nor in the sensitivity calculation. The injection pseudo-measurement at the highvoltage side of the distribution transformer is the power flow in that transformer.

Finally, zero injections yield constraints (8) at buses: 1014, 1021, 4011, 4012, 4021, 4022, 4031, 4032, 4044 and 4045.

The state reconstruction optimization problem is solved on each measurement sample, i.e. every $100 \mathrm{~ms}$. The constrained optimization problem is solved by applying Newton method to the Karush-Kuhn-Tucker conditions [19]. Furthermore, the reference power injections considered in (5) are the values obtained from the previous state reconstruction execution. The very first state reconstruction uses the pre-disturbance powers.

\section{Sensitivity validation in the absence of measurement noise}

The results presented in this sub-section have been obtained without noise on the simulated measurements, nor on the initial bus power pseudo-measurements.

The long-term unstable evolution of the voltage a bus 1041 is shown in Fig. 3. This bus is located in the Central region (see Fig. 2), which is the region of interest since it experiences dramatic voltage drops. Within that region, bus 1041 is the most impacted.
The same figure shows the evolution of the reconstructed voltage magnitude; in fact the two curves are indiscernible at the chosen scale.

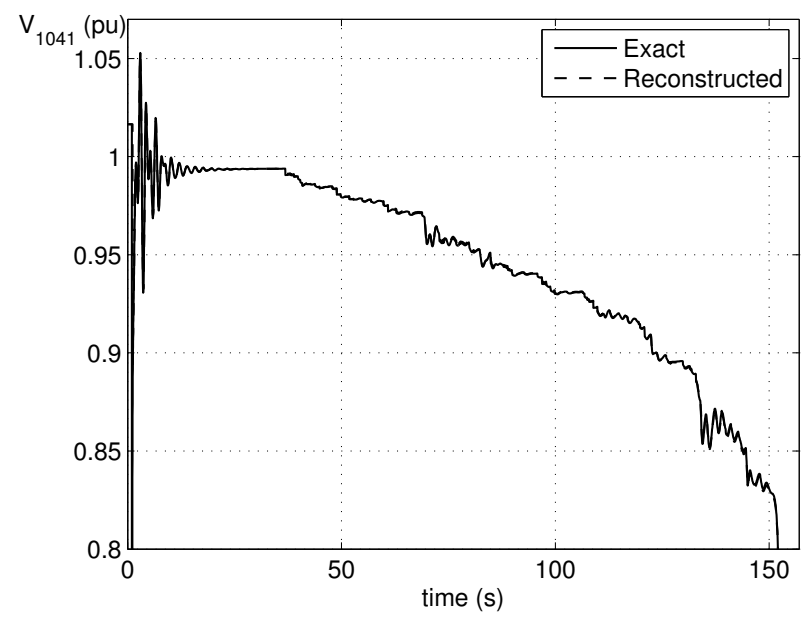

Fig. 3. Voltage magnitude at bus 1041

A closer look at reconstruction errors is provided by Fig. 4, showing the difference between the exact and the reconstructed voltage magnitude. For bus 1041, the error is indeed very small. With a lower rate of reconstruction, errors are slightly larger [14] but the overall accuracy remains very good. For bus 1011, the error is more important but this bus is located far outside the region of interest. Its reconstructed bus voltage is used in the computation of the Jacobian $\varphi_{\mathrm{z}}$ but the relatively larger inaccuracy has no consequence, as shown in the next results.

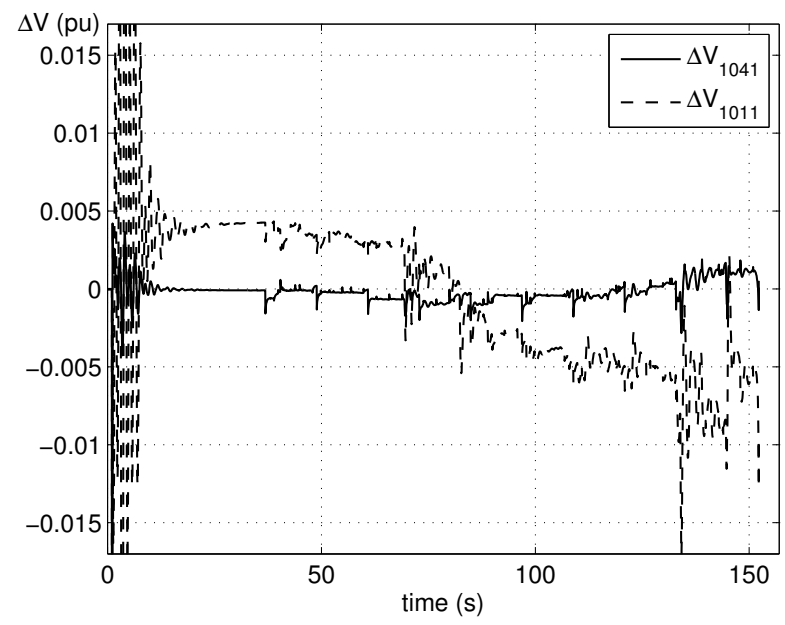

Fig. 4. Reconstruction error at two buses

The evolution of the sensitivity of the total reactive generation to the reactive load at bus 1041 (i.e. the component of $S_{Q_{g} \text { q }}$ relative to that bus) is shown in Fig 5. The figure shows the sensitivities computed from respectively the exact voltages provided by time-domain simulation and the reconstructed voltages. The figure focuses on the time interval where small 


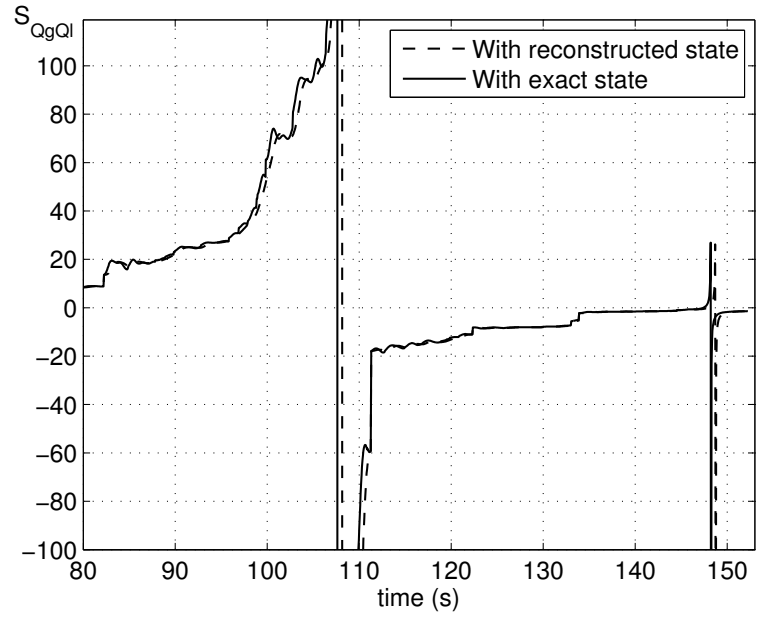

Fig. 5. Sensitivity $S_{Q g Q l}$ at bus 1041

differences are observed between the two curves. In ideal conditions, i.e. using the exact bus voltages, the sensitivity changes sign at $t=107.6 \mathrm{~s}$. When using reconstructed bus voltages, very slightly different values of the sensitivities are obtained, and the change of sign takes place at $t=108.1 \mathrm{~s}$. This delay of $0.5 \mathrm{~s}$ in instability detection when using the reconstructed states is clearly negligible. In both cases, the developing instability is unambiguously detected some $50 \mathrm{~s}$ before the final system collapse. Note that earlier detection can be obtained by anticipating the OEL activation as recalled in Section II.

\section{Impact of PMU configuration}

The next results illustrate the impact of the PMU configuration used for state reconstruction.

As was shown in [14], the 5-PMU configuration is rich enough to accurately track the changing state (typically bus voltages, branch power flows and bus power injections) of the region of interest. In this configuration, generators g11 and g12 are not provided with PMUs. The reconstructed voltage of generator g12 is shown with dashed line in Fig. 6. A comparison with the exact state (solid line in the same figure) shows a somewhat inaccurate reconstruction, with the switching from AVR to OEL control at $t \simeq 77$ s not captured. This is to be expected in so far as g12 is outside the region provided with PMUs.

Figure 7 shows the evolution of the sensitivity $S_{Q g Q l}$ at bus 1041 computed from the reconstructed states using the same 5-PMU configuration. This is to be compared with Fig. 5, obtained with additional PMUs at buses g11 and g12. Without these two PMUs, the instability detection takes place 3.7 seconds later. Although this delay is not critical (anticipation capabilities are still good!), the results suggest that proper sensitivity computation may require to provide with PMUs some more generators than those sufficient to monitor the state of the area impacted by instability. The additional generators are basically those involved in the voltage instability mechanism, such as g11 and g12, whose field currents get limited.

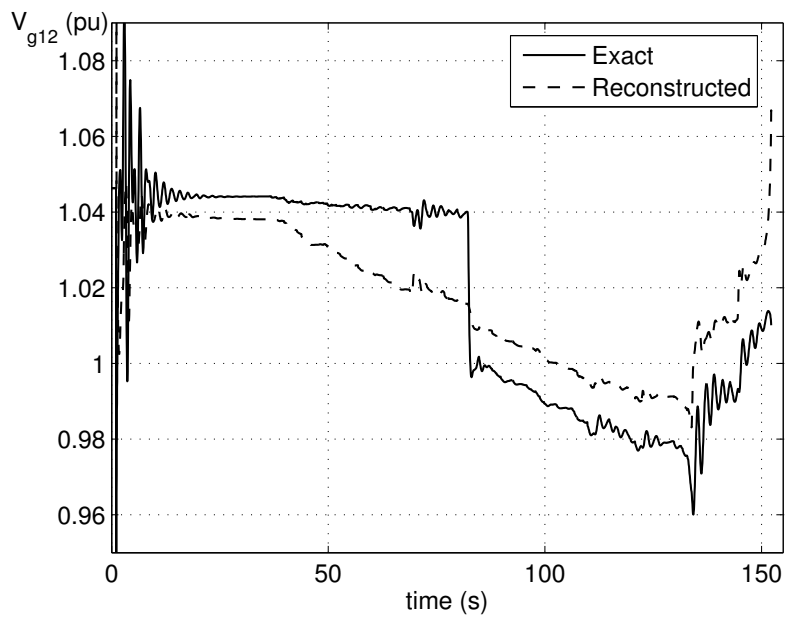

Fig. 6. Voltage magnitude at generator g12

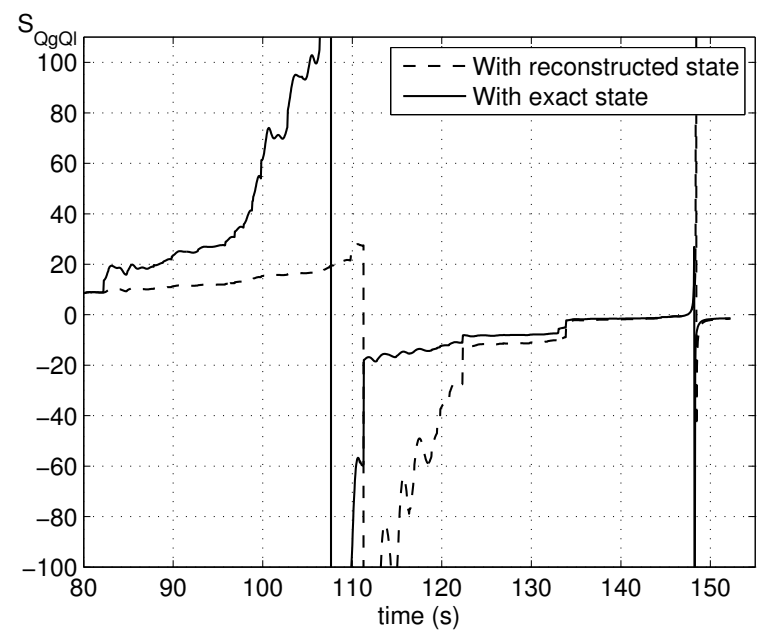

Fig. 7. Sensitivity $S_{Q g Q l}$ at bus 1041, obtained from 5 PMUs

The results presented in the remaining of the paper have been obtained with the 7-PMU configuration.

\section{E. Results with noise on phasor measurements and initial powers}

The more realistic results shown in this section have been obtained with random noise added to the input data used by state reconstruction. Two situations have been considered:

A. a zero-mean, Gaussian noise has been added independently to each $v_{x}, v_{y}, i_{x}$ and $i_{y}$ component relative to a PMU. The standard deviation of this noise has been set to $0.003 \mathrm{pu}$, as suggested in $[16]^{3}$;

B. in addition, the bus power pseudo-measurements used in the first state reconstruction have been obtained by adding a zero-mean Gaussian noise with standard deviation of $0.01 \mathrm{pu}$ to the exact values. This represents the error affecting the bus powers stemming from the classical state estimator.

${ }^{3}$ thus, the noise lies in $[-0.01+0.01]$ pu with $99.9 \%$ probability 
The weight $w_{P j}$ or $w_{Q j}$ assigned to a phasor measurement is set to the inverse of the noise variance. The standard deviation of pseudo-measurements is assumed to be 10 times larger than that of phasor measurements, thus leading to weights $w_{i} 100$ times smaller.

A zoom on the evolution of voltage at bus 1041, when the most important changes are taking place, is shown in Fig. 8, comparing the exact (solid line) with the reconstructed (dotted line) voltage. It is seen that the latter fluctuates around the former with an acceptable deviation in the order of $0.01 \mathrm{pu}$.

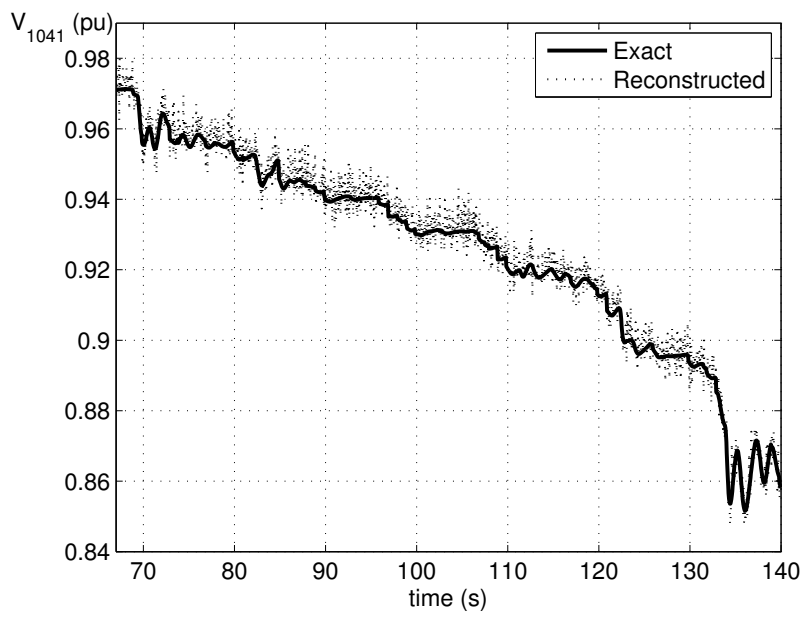

Fig. 8. Voltage magnitude at bus 1041 in presence of measurement noise

The corresponding sensitivity evolutions are shown in Fig. 9. Note that, according to the procedure detailed in [2], the reconstructed voltages have been post-processed with a simple moving average filter before being used in the sensitivity computation, as indicated in Fig. 1. In [2] this was shown to yield much better results than filtering the sensitivities, i.e. placing the filter at point 1 in the flowchart of Fig. 1. Alternatively, one could think of filtering the input phasor measurements, i.e. placing the filter at point 2 in the flowchart. According to our tests, this yields significantly worse results. One reason is that the moving average filter somewhat "desynchronize" the phasor measurements, and introduces some sort of time skew.

Figure 9 shows that the moving average filter used to filter the reconstructed voltages introduces a small delay of $3.6 \mathrm{~s}$ in instability detection, but the overall sensitivity evolution is unchanged. The dashed and dash-dotted curves in the figure refer to the noisy data variants $\mathrm{A}$ and $\mathrm{B}$, respectively. In the shown time interval, the two curves are indiscernible. In fact, they slightly differ for the first state reconstructions, when the effect of the initial pseudo-measurements is still felt. Since each state reconstruction relies on the bus injections computed by the previous reconstruction, the effect of the initial error on pseudo-measurements dies out as time passes.

\section{CONCLUSION}

This paper has considered the applicability of the widearea voltage instability detection method introduced in [1], [2]

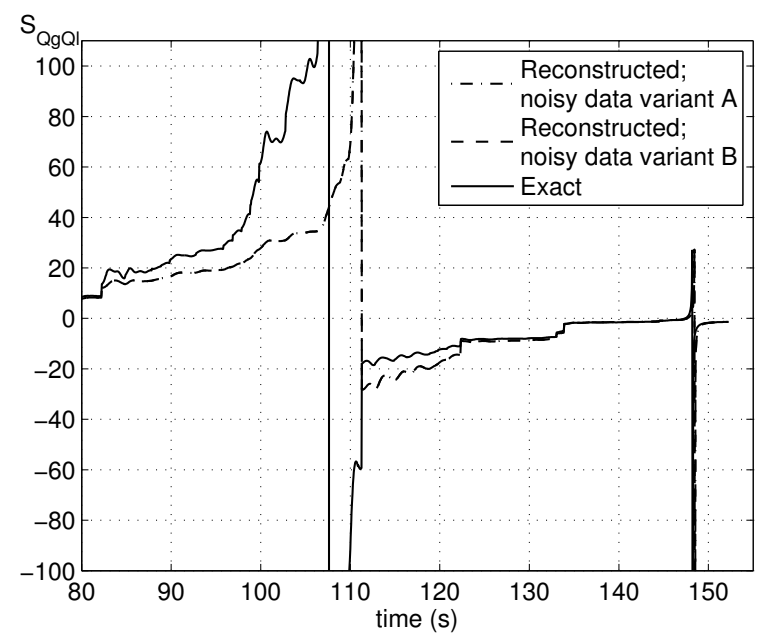

Fig. 9. Sensitivity $S_{Q g Q l}$ at bus 1041 in presence of measurement noise

when a limited number of PMUs is available, which will be the prevailing situation still for quite some time. Simply stated, the sensitivity-based detection method is unchanged, while the complex bus voltages required to compute those sensitivities are provided by the state reconstruction algorithm proposed in [13], [14].

The results have been obtained with synchronous phasor measurements simulated from the outputs of a detailed time simulation. A small but realistic test system experiencing longterm voltage instability has been considered.

These results clearly indicate that the sensitivity-based method is still capable of unambiguously detecting an impending voltage instability. With respect to a mere monitoring of voltages, it allows anticipating the degradation of system operating conditions. Conversely, the results confirm the performances of the state reconstruction method in a demanding application such as voltage instability detection through sensitivity computation.

Resorting to state reconstruction makes the method of [1], [2] scalable, i.e. it is able to accommodate different PMU measurement configurations, starting with PMUs located at key generator buses and progressing in some farther future towards full observability of the monitored region.

\section{REFERENCES}

[1] M. Glavic, T. Van Cutsem, "Wide-Area Detection of Voltage Instability From Synchronized Phasor Measurements. Part I: Principle," IEEE Trans. Power Syst., Vol. 24, No. 3, pp. 1408-1416, Aug. 2009

[2] M. Glavic, T. Van Cutsem, "Wide-Area Detection of Voltage Instability From Synchronized Phasor Measurements. Part II: Simulation Results," IEEE Trans. Power Syst., Vol. 24, No. 3, pp. 1417-1425, Aug. 2009

[3] M. Glavic, T. Van Cutsem, "A short Survey of Methods for Voltage Instability Detection," in Proc. IEEE PES General Meeting, Detroit, MI, July 2011

[4] A. G. Phadke, J. S. Thorp, Synchronized Phasor Measurements and Their Applications, Springer, 2008

[5] M. M. Begovic, A. G. Phadke, "Voltage Stability Assessment Through Measurement of a Reduced State Vector," IEEE Trans. Power Syst., Vol. 5, No. 1, pp. 198-203, Feb. 1990

[6] C. Cañizares (Editor/Coordinator), Voltage Stability Assessment: Concepts, Practices, and Tools, IEEE PES publication, Power System Stability Subcommittee, ISBN 0780378695, 2002. 
[7] C. W. Taylor, Power System Voltage Stability, EPRI Power System Engineering Series, McGraw Hill, 1994

[8] T. Van Cutsem, C. Vournas, Voltage Stability of Electric Power Systems, Boston, Kluwer Academic Publishers, 1998

[9] R. F. Nuqui, "State Estimation and Voltage Security Monitoring Using Synchronized Phasor Measurements," PhD Thesis, Virginia Polytechnic Institute and State University, Blacksburg, Virginia, USA, Jul. 2001

[10] R. F. Nuqui and A. G. Phadke, "Phasor Measurement Unit Placement for Complete and Incomplete Observability," IEEE Trans. Power Del., Vol. 20, No. 4, pp. 2381-2388, Oct. 2005.

[11] N. Zhou, Z. Huang, J. Nieplocha, T. B. Nguyen, "Wide-Area Situational Awareness of Power Grids with Limited Phasor Measurements," in Proc. Third International Conference on Critical Infrastructures (CRIS), Alexandria, VA, Sept. 2006.

[12] T. Overbye, P. Sauer, C. DeMarco, B. Lesieutre, M. Venkatasubramanian, Using PMU Data to Increase Situational Awareness, Power System Engineering Research Center (PSERC) Publication 10-16, Sept. 2010.

[13] M. Glavic, T. Van Cutsem, "Investigating State Reconstruction from Scarce Synchronized Phasor Measurements," in Proc. IEEE PowerTech,
Trondheim, Norway, June 2011

[14] M. Glavic, T. Van Cutsem, "State Reconstruction from Synchronized Phasor Measurements," in Proc. IEEE Innovative Smart Grid Technology (ISGT) 2011, Manchester, UK, December 2011.

[15] H. Kwakernaak, R. Sivan, Linear Optimal Control Systems, John Wiley \& Sons, Inc., 1972

[16] A. P. Meliopoulos (Task Force Leader), Synchrophasor Measurement Accuracy Characterization, North American SynchroPhasor Initiative (NASPI) Interim Report (Performance and Standards Task Team), Aug. 2007.

[17] S. Chakrabarti and E. Kyriakides, "PMU Measurement Uncertainty Considerations in WLS State Estimation," IEEE Trans. on Power Syst., vol. 24, no. 2, pp. 1062-1071, May 2009.

[18] M. Glavic, T. Van Cutsem, "Adaptive Wide-Area Closed-Loop Undervoltage Load Shedding Using Synchronized Measurements," Proc. 2010 IEEE Power Eng. Soc. General Meeting, Minneapolis (USA), Jul. 2010

[19] D. I. Sun, B. Ashley, B. Brewer, A. Hughes, and W. F. Tinney, "Optimal power flow by Newton approach," IEEE Trans. on PAS, vol. PAS-103, no. 10, pp. 2864-2880, Oct. 1984. 